\title{
Clinico-pathological effects of some herbal extracts: time for a seed change in understanding
}

\author{
Paul Sibbons
}

Published online: 1 November 2014

(C) Springer-Verlag London 2014

Welcome to a significant departure for Comparative Clinical Pathology. We have been stimulated to produce this special issue by the increasing number of well-constructed manuscripts we are now receiving which are investigating changes to or maintenance of clinical pathology indices during experimental treatment with herbal extracts. Some of these manuscripts record positive changes to disease-state indices by the use of herbal extracts and others demonstrate maintenance of normal parameters during experimental induction of disease states. Diabetes, of course, is one of those conditions which is often targeted with the use of herbal extracts, and it is interesting to note that, in some remotely living and isolated tribes, diabetes is an unrecorded condition. Whether this is because of their general lifestyle or whether it reflects a diet containing medicinal herbal extracts is currently unclear, but some of the manuscripts we are now receiving at $\mathrm{CCP}$ are starting to investigate these questions.

Herbal extract remedies (and some other natural substances) are also increasingly being investigated in areas such as wound healing both externally and internally.
In this era of increasing resistance to current antibiotics and the reduction in efficacy of some of our traditional, chemistryfounded treatments, particularly for long-term conditions, investigation of alternatives including historically and currently used herbal remedies is to be encouraged and will no doubt escalate in importance as the world population increases and, concomitantly, our demands for readily available effective treatments increase.

The potential to use natural herbal medicines, natural substances or extracted elements thereof is one of the components in man's armoury against expanding intensity and numbers of both current and future disease conditions affecting both man and animals.

CCP welcomes discussion of this special issue through the standard issues of Comparative Clinical Pathology and may consider further special issues in appropriate areas in the future.

Editor in chief

Professor Paul Sibbons
P. Sibbons $(\bowtie)$

Department of Surgical Research, Northwick Park Institute of

Medical Research, Harrow HA1 3UJ, UK

e-mail: p.sibbons@ucl.ac.uk 